DOI: 10.12731/2218-7405-2018-4-49-63

УДК 1(159.9)

\title{
РАЗВИТИЕ КОМПОНЕНТОВ ЭМОЦИОНАЛЬНОГО ИНТЕЛЛЕКТА ОПЕРАТОРОВ КОНТАКТНОГО ЦЕНТРА
}

\author{
Судакова Е.Н.
}

Цель. Основу статьи составляют результаты экспериментального исследования влияния краткосрочного тренинга развития эмоционального интеллекта на рост уровня компонентов ЭИ у операторов контактного ичентра.

Метод и методология проведения работы. В исследовании участвовали 53 оператора контактного цуентра телекоммуникационной компании: 23 человека составили экспериментальную группу и прошли тренинг, направленный на развитие эмоционального интеллекта, 30 человек составили контрольную группу. Группь не отличались по возрасту и среднему стажу работы. Для определения уровня эмоционального интеллекта использовался опросник «ЭмИн» Д.В. Люсина.

Результаты исследования не выявили статистически значимых изменений в компонентах эмоционального интеллекта. Качественный анализ проявил некоторые особенности восприятия эмоций у участников экспериментальной группь: средние и максимальные значения компонентов ЭИ в экспериментальной группе увеличились после тренинга, в интервью участники отметили, что стали обращать больще внимания на свои эмочии и эмоции окружающих.

Область применения результатов. Результаты могут быть применены при планировании обучения и развития сотрудников контактных иентров. Развитие эмоционального интеллекта сотрудников на рабочем месте формирует лучшее понимания собственных эмочий, эмоций клиентов и коллег, что может способствовать повышению их эмоционального благополучия.

Ключевые слова: эмоциональный интеллект; операторы контактного ичентра; понимание эмоций; управление эмоциями. 


\section{THE PROBABILITY OF THE DEVELOPMENT OF CALL CENTER OPERATOR'S EMOTIONAL INTELLIGENCE}

\section{Sudakova E.N.}

The aim of the study was to determine the effect of training on the development of emotional intelligence of the contact center operators. The study involved 53 operators of Telecom Company: 23 people accounted for the experimental group participated in training supposed to develop their emotional intelligence, 30 people were in the control group and didn t take part in training. Groups did not differ in age and average length of work experience. To determine the level of emotional intelligence before and after the training we used questionnaire "EmIn» by D.V. Lyusin. The results of the study did not show any changes in the competence of emotional intelligence. Qualitative analysis showed some features of the perception of emotions in the participants of the experimental group. Developing the emotional intelligence of employees in the workplace creates a better understanding of one's own emotions, the emotions of clients and colleagues, which can enhance their emotional well-being.

Keywords: emotional intelligence; contact center operators; understanding emotions; managing emotions.

\section{Введение}

Контактный центр - это организация или подразделение, куда направляются звонки от клиентов компании. Актуальность данного исследования связана с тем, что сейчас система контактных центров (КЦ) переживает значительный рост. Значимость контактных центров становится необыкновенно высокой, в их задачи входят обоснованные рекомендации, психологическая поддержка, сохранение и повышение лояльности клиентов. В связи с этим растут требования к уровню профессионализма операторов КЦ, в частности к их умению взаимодействовать с клиентами по конфликтным вопросам. 
В течение дня оператор принимает порядка 200 звонков. Такая нагрузка сочетается с рядом ограничений: короткое время диалога, необходимость отвечать на претензии и недовольства, невозможность проявления спонтанной эмоциональной реакции на слова и действия клиентов, регламентированные перерывы, отсутствие закрепленного рабочего места, высокая степень контроля со стороны организации $[1 ; 3 ; 6]$. Сами операторы часто описывают свою работу как скучную, напряженную, связанную с высоким уровнем стресса $[6 ; 10]$. Неспособность оператора справляться с эмоциональной нагрузкой отрицательно влияет на качество выполнения его задач.

Это означает, что кроме высокой работоспособности, навыков работы с программным обеспечением и знания продуктов и регламентов компании, оператор должен уметь верно определять эмоции клиента во время разговора, уменьшать негативные чувства клиента по отношению к компании и приводить диалог к завершению.

Концепция эмоционального интеллекта (ЭИ) полезна для операторов контактных центров, так как позволяет определить необходимые для эффективной работы эмоциональные навыки - понимание эмоций и управление как своими эмоциями, так и эмоциями других людей. В данном исследовании мы опирались на концепцию ЭИ Д.В. Люсина [2; 4].

Д.В. Люсин предложил собственную модель эмоционального интеллекта. Он определяет ЭИ как «совокупность способностей для понимания своих и чужих эмоций и управления ими», не включая в нее личностные особенности [4].

Эмоциональный интеллект, в концепции Д.В. Люсина, состоит из внутриличностного эмоционального интеллекта (ВЭИ) и межличностного эмоционального интеллекта (МЭИ). И ВЭИ и МЭИ включают в себя два компонента - понимание и управление эмоциями, которые для ВЭИ относятся к внутреннему опыту человека, а для МЭИ - к внешнему миру [2; 4].

Возможность развития ЭИ в рамках профессиональной деятельности позволила бы повысить эффективность работы операторов 
КЦ в конфликтных ситуациях, снизить их уровень стресса, увеличить продуктивность [6].

Однако, не существует однозначных доказательств того, что компоненты эмоционального интеллекта могут быть развиты при помощи тренингов. Так, исследование учителей, проведенное P. Коркоран и P. Тормей (Corcoran R., Tormey R.), не показало разницы среднего уровня ЭИ между контрольной и экспериментальной группой после краткосрочного тренинга, который состоял из 6 встреч. В то же время наблюдалось статистически незначимое увеличение средних значений компонентов ЭИ у участников тренинга [7].

В работе C. Херпертс (Herpertz S.) у испытуемых после тренинга значимо увеличилась способность к распознаванию эмоций по лицам (по результатам теста MSCEIT), при этом результаты определения эмоций по изображениям не возросли [9]. Ряд других исследований показали рост среднего показателя ЭИ после тренинга [5; $8 ; 11 ; 12]$. Долгосрочные программы показывают результаты [11], однако, их применение в работе контактных центров маловероятно, по причине их длительности.

H. Шутте (Schutte N.) говорит о недостаточности исследований, связанных с развитием компонентов эмоционального интеллекта, и преимуществах, которые получают испытуемые в результате их развития $[13 ; 14 ; 15]$.

Эмоциональный интеллект положительно влияет на эффективность работы оператора, в связи с этим актуально изучить возможности развития компонентов ЭИ в короткие сроки. Исследование направлено на то, чтобы понять на примере операторов КЦ, насколько краткосрочные программы, ориентированные на развитие эмоционального интеллекта, способны влиять на изменение уровня ЭИ.

Целью данной работы стало исследование влияния краткосрочного тренинга развития ЭИ на его уровень и оценка компонентов эмоционального интеллекта до и после тренинга. В основу исследования легла модель ЭИ Д.В. Люсина. 


\section{Материалы и методы}

Эмпирической базой исследования выступил контактный центр телекоммуникационной компании.

Объектом исследования стали операторы контактного центра. Общий объем выборки составил 53 человека, 23 оператора в экспериментальной группе и 30 человек в контрольной.

Средний возраст участников экспериментальной группы - 31 год, 19 участников женщины, 4 - мужчины. Такое распределение связано с тем, что сотрудниками контактных центров преимущественно являются женщины. Средний стаж работы испытуемых в контактном центре - 41 месяц. Контрольная группа также преимущественно состояла из женщин - 26 человек, средний возраст $-29,6$, средний стаж работы - 39 месяцев.

Демографическое сравнение экспериментальной и контрольной выборки, проведенное с использованием критерия МаннаУитни, не показало разницы между группами по стажу работы и возрасту.

Для проведения исследования был разработан тренинг, направленный на развитие компонентов эмоционального интеллекта. Особенностью тренинга являлась его краткосрочность, связанная со спецификой работы операторов КЦ и необходимостью получения результатов в максимально короткие сроки. Тренинг включал 5 встреч с периодичностью 1 раз в неделю, по 2 часа.

Цель тренинга была сформулирована как развитие компонентов эмоционального интеллекта операторов контактного центра, в связи с чем были поставлены следующие задачи:

- Развитие навыка распознавания собственных эмоций;

- Развитие способности распознавания эмоций других людей (клиентов и коллег);

- Обучение навыкам управления собственными эмоциями;

- Обучение навыкам влияния на эмоции других людей.

Процесс обучения проходил при поддержке членов группы тренером и другими участниками, что позволяло расширить понимание особенностей проявления и выражения эмоций. 
Тренинг состоял из следующих основных этапов:

1. Понимание важности осознания эмоций в личной и профессиональной жизни, концентрация внимания на том, как эмоции, которые участники тренинга испытывают на работе, влияют на их продуктивность - первая встреча.

2. Расширение эмоционального словарного запаса, осознание собственных эмоций, осознание эмоций других участников тренинга, при постоянной обратной связи - вторая встреча.

3. Работа с агрессивными эмоциями, осознание агрессии и поиск эффективных и безопасных способов ее выражения - третья встреча.

4. Обучение техникам регуляции эмоций, выполнение домашнего задания с целью применения новых навыков - встречи с третьей по пятую.

Каждое занятие включало в себя обсуждение актуального эмоционального состояния участников, упражнения, способствующее переключению участников с рабочих задач на обучающий процесс, теоретическую часть, по теме ЭИ, упражнения, направленные на развитие способностей ЭИ, шеринг.

В исследовании применялись следующие методы:

1. Эмпирические методы - опросник и интервью. Методики исследования: опросник ЭмИн, Д.В. Люсина, интервью на основе модели ЭИ Д.В. Люсина.

2. Математико-статистические методы обработки данных: критерий Манна-Уитни, критерий Вилкоксона. Обработка данных проводилась при помощи пакета прикладных программ статистической обработки данных SPSS 17.00.

На рис. 1 отображена схема проведения исследования. Обе группы испытуемых заполнили опросник ЭмИн на определение уровня эмоционального интеллекта до и после тренинга. Экспериментальная группа помимо прохождения тренинга также участвовала в посттренинговом интервью. В интервью были рассмотрены компоненты ЭИ, выделенные Д.В. Люсиным [9], также задавались вопросы об эмоциональном опыте участников тренинга. 


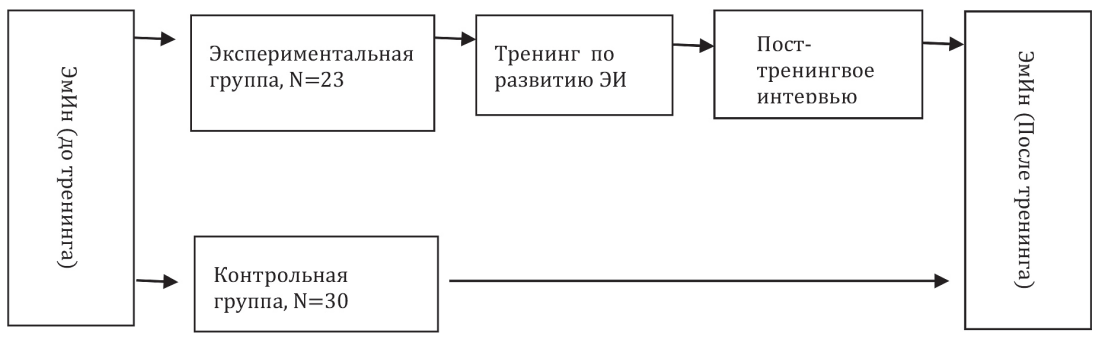

Рис. 1. Схема исследования

\section{Результаты исследования}

Коэффициент внутренней согласованности (альфа Кронбаха) для методики «Эмоциональный интеллект» составил 0,916\%, что позволяет рассматривать результаты исследования как достоверные.

В связи с тем, что экспериментальная группа составила 23 человека, для проверки нормальности распределения признаков был использован критерий Вилкоксона. Анализ показал, что в экспериментальной группе результаты первого измерения МЭИ, ВЭИ, ПЭ распределены нормально, УЭ не распределен нормально. При проведении второго измерения, ни один из компонентов эмоционального интеллекта не распределен нормально. В контрольной группе $(\mathrm{N}=30)$ в пре- и посттренинговом исследовании, ни один из компонентов ЭИ не распределен нормально. В связи с этим, для сравнения выборок использовался критерий Вилкоксона.

В табл. 1 отражены средние значения и стандартное отклонение для итогового значения эмоционального интеллекта для экспериментальной и контрольной группы до и после тренинга. Среднее значение общего уровня эмоционального интеллекта увеличилось незначительно, с 97,5 до 101,6, в то время как в контрольной группе среднее значение общего уровня ЭИ уменьшилось, с 96,04 до 95,4.

Сравнения компонентов ЭИ до и после тренинга в экспериментальной и контрольной группах, с использованием критерия Вилкоксона, показали отсутствие значимых изменений в результате краткосрочного тренинга. 
Таблицуа 1.

Сравнение средних значений и стандартного отклонения общего уровня ЭИ до и после тренинга в экспериментальной и контрольной группах

\begin{tabular}{|c|c|c|}
\hline Группа & $\begin{array}{c}\text { Среднее значение } \\
\text { уровня ЭИ до тренинга }\end{array}$ & $\begin{array}{c}\text { Среднее значение ЭИ } \\
\text { после тренинга }\end{array}$ \\
\hline Экспериментальная & 97,5 & 101,6 \\
группа & $\sigma=16,03$ & $\sigma=19,35$ \\
\hline \multirow{2}{*}{ Контрольная группа } & 96,04 & 95,4 \\
& $\sigma=16,84$ & $\sigma=16,24$ \\
\hline
\end{tabular}

В табл. 2 приведены результаты сравнения компонентов эмоционального интеллекта до и после тренинга. Из таблицы видно, что значимые различия в результатах исследования компонентов эмоционального интеллекта у участников экспериментальной группы отсутствуют.

Таблицуа 2.

Пре- и посттрениновое сравнение компонентов EQ участников экспериментальной группы

\begin{tabular}{|c|c|c|c|c|c|c|}
\hline & \multicolumn{2}{|c|}{ До тренинга } & \multicolumn{2}{c|}{ После тренинга } & \multirow{2}{*}{ sig } & \multirow{2}{*}{ p } \\
\cline { 2 - 4 } & Mean & SD & Mean & SD & & 0,145 \\
\hline МЭИ & 47,1 & 9,58 & 49 & 10,73 & 0,05 \\
\hline ВЭИ & 47,9 & 9,29 & 49 & 11,14 & 0,406 & 0,05 \\
\hline ПЭ & 44,8 & 8,15 & 48,9 & 9,98 & 0,145 & 0,05 \\
\hline УЭ & 46,8 & 10,22 & 49,1 & 12,1 & 0,347 & 0,05 \\
\hline
\end{tabular}

Таблица 3.

Пре- и посттренинговое сравнение компонентов ЭИ участников контрольной группы

\begin{tabular}{|c|c|c|c|c|c|c|}
\hline \multirow{2}{*}{} & \multicolumn{2}{|c|}{ До тренинга } & \multicolumn{2}{|c|}{ После тренинга } & \multirow{2}{*}{ sig } & \multirow{2}{*}{ p } \\
\cline { 2 - 5 } & Mеan & SD & Mean & SD & & \\
\hline МЭИ & 46,65 & 8,82 & 46,47 & 8,52 & 0,182 & 0,05 \\
\hline ВЭИ & 49,39 & 8,74 & 48,91 & 8,64 & 0,638 & 0,05 \\
\hline ПЭ & 46,73 & 8,51 & 46,73 & 7,6 & 0,124 & 0,05 \\
\hline УЭ & 49,3 & 8,71 & 49,3 & 8,8 & 0,961 & 0,05 \\
\hline
\end{tabular}

Эксперимент проходил на протяжении пяти недель. Обе группы и экспериментальная, и контрольная продолжали работать и подвергались типичному для них уровню стресса. Неизменность характеристик показателей ЭИ у участников контрольной группы 
(рис. 2) говорит о том, что ЭИ стабильная характеристика, которая изменяется с трудом. При этом даже незначительные изменения компонентов ЭИ для экспериментальной группы говорят о возможном влиянии тренинга на экспериментальную группу

На рисунке 2 продемонстрированы изменения в распределении и значений средних у компонентов эмоционального интеллекта до и после тренинга. Так, из рис. 2 видно, что в экспериментальной группе средние значения после тренинга возросли, в то время как в контрольной группе они остались неизменными или незначительно уменьшились.
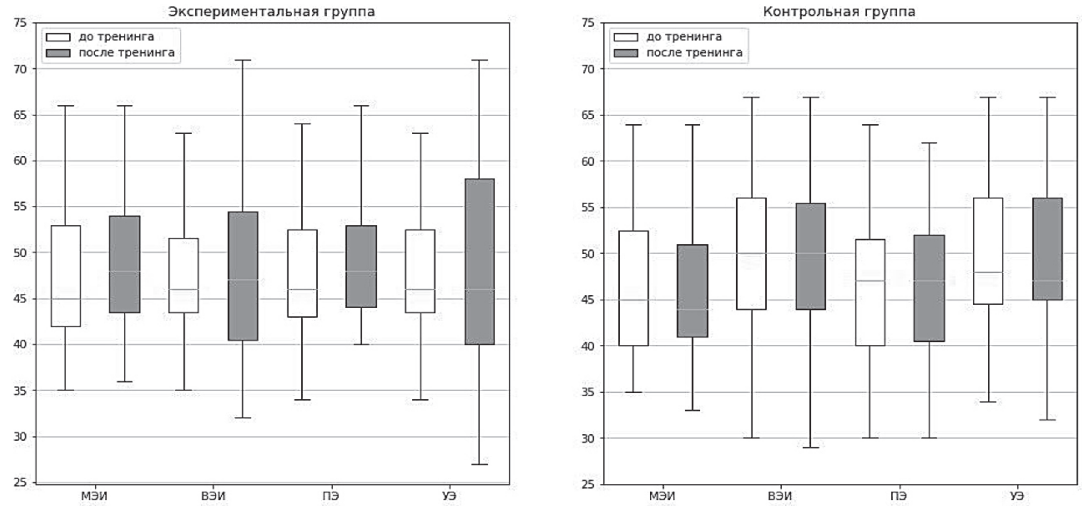

Рис. 2. Сравнение компонентов ЭИ участников экспериментальной группы до и после тренинга

На рис. 2 отображены изменения в средних, 25 и 75 процентилях, минимальных и максимальных значениях в группах до и после тренинга. Рисунок позволяет нам оценить изменения, произошедшие в выборках, но которые не могут быть отображены при сравнении с использованием критерия Вилкоксона.

В экспериментальной группе возросли максимальные значения всех компонентов ЭИ, в то время как в контрольной группе максимальное значение практически не изменилось или уменьшилось.

В экспериментальной группе значения ВЭИ и УЭ минимальное значение стало еще меньше, в контрольной группе нет большой разницы между минимальными значениями до и после тренинга. 
Для углубления понимания влияния тренинга на участников, с ними было проведено интервью, вопросы которого строились вокруг компонентов ЭИ, выделенных Д. Люсиным [4]. Обобщенные результаты интервью представлены в таблице 4.

Данное интервью проявило некоторые аспекты воздействия тренинга на участников, которые не могли быть зафиксированы при помощи опросника, но были озвучены испытуемыми.

Так, участники экспериментальной группы стали обращать больше внимания на собственные эмоции, увидели взаимосвязь между собственными чувствами и поведением. Кроме того, они стали замечать и считать значимыми эмоции клиентов и коллег, а также учитывать эту информацию в ежедневном взаимодействии.

Ряд участников тренинга заметили, что они часто неверно интерпретируют эмоции других людей, в процессе тренинга им удавалось получать обратную связь. Для них стали очевидны собственные ошибки в понимании эмоций других людей.

Два участника контрольной группы заметили, что после тренинга они подвергли сомнению свою уверенность в том, что им всегда хорошо удается управлять собственными эмоциями и понимать их. Возможно, рост критичности и стал причиной уменьшения минимальных значений компонентов «Внутриличностный эмоциональный интеллект» и «Управление эмоциями» в контрольной группе испытуемых после тренинга.

Таблица 4.

Обобщенные данные итогового интервью с участниками экспериментальной группы

\begin{tabular}{|c|c|c|}
\hline $\begin{array}{c}\text { Количество от } \\
\text { общего числа } \\
\text { опрошенных }\end{array}$ & $\begin{array}{c}\text { Обобщение по ответам } \\
\text { интервью }\end{array}$ & Пример высказывания \\
\hline $39 \%$ & $\begin{array}{l}\text { Улучшение понимания } \\
\text { собственных эмоций. } \\
\text { Рост внимания к своим } \\
\text { чувствам }\end{array}$ & $\begin{array}{l}\text { «Стараюсь все-таки выдохнуть и по- } \\
\text { нять, что я осознаю здесь и сейчас, } \\
\text { какие чувство это у меня вызывает, } \\
\text { важно это для меня или нет, а только } \\
\text { после этого принимать решения, го- } \\
\text { ворить». }\end{array}$ \\
\hline
\end{tabular}


Окончание табл. 4.

\begin{tabular}{|c|c|c|}
\hline $30,4 \%$ & $\begin{array}{l}\text { Улучшение способно- } \\
\text { сти опознавать чувства } \\
\text { других людей. Интерес } \\
\text { к чувствам других. }\end{array}$ & $\begin{array}{l}\text { «Я стала более внимательно относить- } \\
\text { ся к чувствам своих коллег и клиен- } \\
\text { тов, хотя раньше я придерживалась } \\
\text { жесткой позиции». }\end{array}$ \\
\hline $65 \%$ & $\begin{array}{l}\text { Использование спо- } \\
\text { собов регулирования } \\
\text { собственного эмоцио- } \\
\text { нального состояния }\end{array}$ & $\begin{array}{l}\text { «При разговоре с конфликтным кли- } \\
\text { ентом я рисую, слежу за своим дыха- } \\
\text { нием, концентрируюсь на телесных } \\
\text { ощущениях» }\end{array}$ \\
\hline $39 \%$ & $\begin{array}{l}\text { Использование спосо- } \\
\text { бов влияния на эмоции } \\
\text { других людей }\end{array}$ & $\begin{array}{l}\text { «Теперь я понимаю, что в разговоре } \\
\text { лучше ориентировать клиента на бу- } \\
\text { дущее, чем оправдываться или делать } \\
\text { вид, что не замечаю, что мне грубят» }\end{array}$ \\
\hline
\end{tabular}

Из вышесказанного видно, что интервью явилось важным дополнением к пониманию результатов тренинга для участников.

\section{Обсуждение результатов}

Обобщим полученные результаты:

Для проведения исследования ЭИ был разработан краткосрочный, ориентированный на специфику работы операторов контактного центра, тренинг.

Анализ до- и пост-тренинговых данных демонстрирует отсутствие статистически значимых изменений в уровне развития компонентов эмоционального интеллекта после проведения всех встреч. Это говорит о том, что уровень эмоционального интеллекта остается стабильной характеристикой даже при наличии внешнего воздействия.

В то же время, качественный анализ обозначил важные отличия: мы наблюдаем рост средних и максимальных значений компонентов ЭИ после тренинга. Обратная связь, полученная от участников, зафиксировала рост интереса к собственным эмоциям и эмоциям других людей, а также готовность использовать навыки регулирования собственного эмоционального состояния. Это, несомненно, является важным результатом в профессиональной деятельности операторов контактных центров.

\section{Выводы}

Получается, за короткий срок сложно статистически уловить изменения в составляющих эмоционального интеллекта, однако про- 
веденное интервью показало, что участники почувствовали рост компетентности в понимании собственной эмоциональной сферы, что согласуется с данными Р. Коркорана (Corcoran R.) [7], C. Херпертс (Herpertz S.) [9]. Проведение тренингов положительно влияет на самопонимание сотрудников, при этом для развития эмоционального интеллекта в долгосрочных программах, требуются изменения в подходе к процессу обучения и развития сотрудников контактных центров.

Полагаем, для оценки изменения компонентов ЭИ требуется более детальный анализ изменений статистических характеристик выборки, также необходимо формулировать дополнительные критерии влияния тренингов.

\section{Список литературы}

1. Бочкарева Е.Н. Компоненты выгорания и стратегия поведения в стрессовых ситуациях операторов контактного центра // Организационная Психология. 2016а. Т. 6. № 2. С. 75-88.

2. Дегтярёв А.В. «Эмоциональный интеллект»: становление понятия в психологии [Электронный ресурс] // Психологическая наука и образование psyedu.ru. 2012. №2. URL: http://psyedu.ru/journal/2012/2/2912. phtml (дата обращения: 20.02.2017).

3. Жеглова К.Ю. (2007). Особенности динамики формирования психического выгорания в профессии оператора телекоммуникационной связи: дисс. ... канд. психол. наук. Ярославль. 2007. 167 с.

4. Люсин Д.В., Прихидько А.И., Гулевич О.А. Новая методика для измерения эмоционального интеллекта: опросник ЭмИн // Психологическая Диагностика. 2006. №4. С. 3-22.

5. Хлевная Е.А., Штроо В.А., Киселева Т.С. Экспериментальное исследование возможности развития эмоционального интеллекта [Электронный ресурс] // Психологическая наука и образование psyedu. ru. 2012. №3. URL: http://psyedu.ru/journal/2012/3/3018.phtml (дата обращения: 02.02.2017).

6. Chambel M.J., Castanheira F. Training opportunities and employee exhaustion in call centres: mediation by psychological contract fulfilment // International Journal of Training and Development. 2012. Vol. 16. № 2 . p. 107-117. doi:10.1111/j.1468-2419.2011.00394.x 
7. Corcoran R., Tormey R. Teacher education, emotional competencies and development education // Procedia-Social and Behavioral Sciences. 2010. Vol. 2. № 2. p. 2448-2457. doi: 10.1016/j.sbspro.2010.03.352

8. Crombie D., Lombard C., Noakes T. Increasing emotional intelligence in cricketers: An intervention study // International Journal of Sports Science and Coaching. 2011. Vol. 6. № 1. p. 69-86 doi: 10.1260/1747-9541.6.1.69

9. Herpertz S., Schütz A., Nezlek J. Enhancing emotion perception, a fundamental component of emotional intelligence: Using multiple-group SEM to evaluate a training program // Personality and Individual Differences. 2016. Vol. 95. p. 11-19. doi: 10.1016/j.paid.2016.02.015

10. Hu Q. et al. «East is East and West is West and never the twain shall meet»: work engagement and workaholism across eastern and western cultures // Journal of Behavioral and Social Sciences. 2014. Vol. 1, pp. 6-24.

11. Fletcher I. [и др.]. A pilot study assessing emotional intelligence training and communication skills with 3rd year medical students // Patient Education and Counseling. 2009. № 3 (76). C. 376-379.

12. Reuben E., Sapienza P., Zingales L. Can we teach emotional intelligence // Columbia Business School Research Paper, 2009. doi: 10.2139/ssrn.1915471

13. Schutte N.S. и др. A meta-analytic investigation of the relationship between emotional intelligence and health // Personality and Individual Differences, 2007. Vol. 42. No 6. p. 921-933. doi: 10.1016/j.paid.2006.09.003

14. Schutte N.S., Malouff J.M. Increasing Emotional Intelligence through Training: Current Status and Future Directions // The international journal of emotional education, 2013. Vol. 5. No.1. pp. 56-72.

15. Schutte N.S., Malouff J.M. Comment on Developments in Trait Emotional Intelligence Research: A Broad Perspective on Trait Emotional Intelligence // Emotion Review, 2016. Vol. 8. No 4. p. 343-344. doi: $10.1177 / 1754073916650499$

\section{References}

1. Bochkareva E.N. Komponenty vygoraniya i strategiya povedeniya v stressovykh situatsiyakh operatorov kontaktnogo tsentra [Components of burnout and coping strategies among call centre operators]. Organizatsionnaya Psikhologiya [Organizational psychology]. 2016a, no. 2. pp. 75-88. (In Russ., abstr. in Engl.). 
2. Degtyarev A.V. «Emotsional'nyi intellekt»: stanovlenie ponyatiya V psikhologii [Elektronnyi resurs] [Emotional intelligence: formation of the notion in psychology]. Psikhologicheskaya nauka i obrazovanie psyedu.ru. [Psychological Science and Education PSYEDU.ru] 2012, no. 2. Available at: http://psyedu.ru/journal/2012/2/2912.phtml (Accessed: 20.02.2017). (In Russ. abstr. in Engl.).

3. Zheglova K.Yu. Osobennosti dinamiki formirovaniya psikhicheskogo vygoraniya $v$ professii operatora telekommunikatsionnoi svyazi [Characteristics of the dynamics of psychic burnout development in profession of wireless communications operator]. Yaroslavl', 2007. 167 p.

4. Lyusin D.V., Prikhid'ko A.I., Gulevich O.A. Novaya metodika dlya izmereniya emotsional'nogo intellekta: oprosnik EmIn [A new technique for measuring emotional intelligence: questionnaire EmIn]. Psikhologicheskaya diagnostika [Psychological Diagnostics]. 2006, no.4, pp. 3-22.

5. Khlevnaya E.A., Shtroo V.A., Kiseleva T.S. Eksperimental'noe issledovanie vozmozhnosti razvitiya emotsional'nogo intellekta [Elektronnyi resurs] [Experimental research of probability of emotional intelligence development]. Psikhologicheskaya nauka i obrazovanie psyedu.ru. [Psychological Science and Education PSYEDU.ru] 2012, no.3. Available at: http://psyedu.ru/journal/2012/3/3018.phtml (Accessed: 02.02.2017). (In Russ. abstr. in Engl.).

6. Chambel M.J., Castanheira F. Training opportunities and employee exhaustion in call centers: mediation by psychological contract fulfillment. International Journal of Training and Development, 2012. V. 16. No. 2. pp. 107-117. doi:10.1111/j.1468-2419.2011.00394.x

7. Corcoran R., Tormey R. Teacher education, emotional competencies and development education. Procedia-Social and Behavioral Sciences, 2010. V. 2. No. 2. pp. 2448-2457. doi: 10.1016/j.sbspro.2010.03.352

8. Crombie D., Lombard C., Noakes T. Increasing emotional intelligence in cricketers: An intervention study. International Journal of Sports Science and Coaching, 2011. V. 6. No. 1. pp. 69-86. doi: 10.1260/1747-9541.6.1.69

9. Herpertz S., Schütz A., Nezlek J. Enhancing emotion perception, a fundamental component of emotional intelligence: Using multiple-group SEM to evaluate a training program. Personality and Individual Differences, 2016. V. 95. pp. 11-19. doi: 10.1016/j.paid.2016.02.015 
10. Hu Q. et al. «East is East and West is West and never the twain shall meet»: work engagement and workaholism across eastern and western cultures. Journal of Behavioral and Social Sciences, 2014. V. 1, pp. 6-24.

11. Fletcher I. A pilot study assessing emotional intelligence training and communication skills with 3rd year medical students. Patient Education and Counseling. 2009. V. 3. No.76, pp. 376-379.

12. Reuben E., Sapienza P., Zingales L. Can we teach emotional intelligence? Columbia Business School Research Paper, 2009. doi: 10.2139/ssrn.1915471

13. Schutte N.S. et al. A meta-analytic investigation of the relationship between emotional intelligence and health. Personal Personality and Individual Differences, 2007. V. 42. No. 6. pp. 921-933. doi: 10.1016/j.paid.2006.09.003

14. Schutte N.S., Malouff J.M. Increasing Emotional Intelligence through Training: Current Status and Future Directions. The international journal of emotional education, 2013. V. 5. No.1, pp. 56-72.

15. Schutte N.S., Malouff J.M. Comment on Developments in Trait Emotional Intelligence Research: A Broad Perspective on Trait Emotional Intelligence. Emotion Review, 2016. V. 8. No. 4. pp. 343-344.doi: $10.1177 / 1754073916650499$

\section{ДАННЫЕ ОБ АВТОРЕ}

Судакова Екатерина Николаевна, ассистент кафедры Психологии и педагогики

Новосибирский Государственный Технический Университет пр-т К.Маркса, 20, г. Новосибирск, Новосибирская область, 630073, Российская Федерация

ekaterina.bochkareva@gmail.com

\section{DATA ABOUT THE AUTHOR}

Sudakova Ekaterina Nikolaevna, Assistant, Department of Psychology and Pedagogy

Novosibirsk State Technical University

20, Karl Marks av., Novosibirsk, 630073, Russian Federation

Ekaterina.bochkareva@gmail.com

SPIN-code: 7579-9404

ORCID: 0000-0001-8927-366X 